\title{
Mechanistic insights into a non-coding risk SNP
}

Studies have previously identified an association between the single nucleotide polymorphism (SNP) rs11672691 at 19q13 - which resides within the long non-coding RNA gene PCAT19 - and aggressive prostate cancer. Two studies now reveal the biological mechanism by which the rs11672691 risk variant promotes aggressive tumour biology.

Gao et al. uncovered an oncogenic regulatory circuit mechanistically underpinning the association between rs11672691 and disease aggressiveness. Initially, SNP genotyping in a cohort of 2,738 men with prostate cancer showed that the rs11672691 G risk allele is associated with advanced $\mathrm{T}$ stage, castration resistance, and PSA progression. Importantly, expression quantitative trait locus (eQTL) analysis in three independent cohorts revealed that the rs11672691 G risk allele is linked to elevated expression of two genes, CEACAM21 and PCAT19. Subsequent knockdown and overexpression experiments in cell lines and gene expression analysis in clinical data sets showed that both genes drive prostate cancer cell growth and metastasis and are upregulated during disease progression, implicating

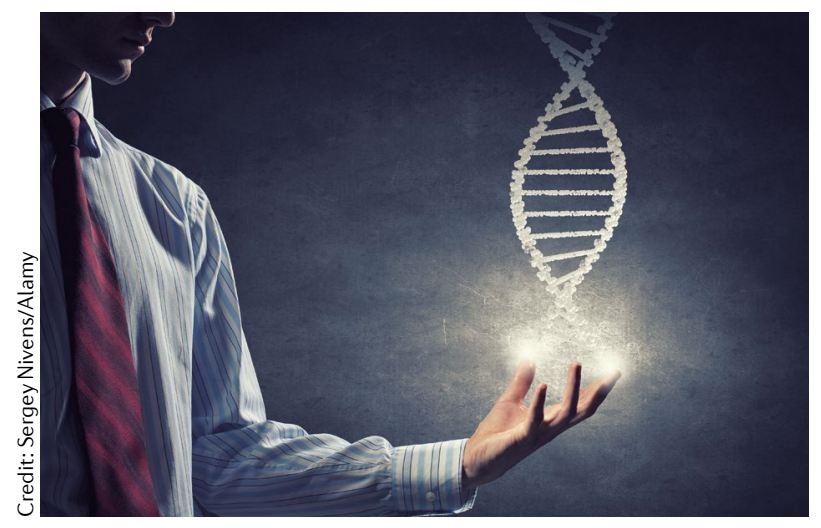

CEACAM21 and PCAT19 as plausible causative genes mediating the effects of rs11672691. Interestingly, rs11672691 mapped to an enhancer element and alters the binding site of homeobox protein HOXA2, a transcription factor associated with poor prognosis. Chromatin looping and genome-editing experiments demonstrated that the rs 11672691 G allele directly drives HOXA2mediated upregulation of PCAT19 and CEACAM21 and promotes aggressive tumour biology.

Additionally, a final clinical correlation analysis uncovered a synergistic effect between the rs11672691 G risk allele and expression of CEACAM21 or PCAT19 on prostate cancer prognosis.

"We plan to validate the diagnostic values in additional large-scale clinical data sets and tumour samples to develop a biomarker for predicting prostate cancer severity and stratifying patients into high-risk and low-risk groups for precision treatment," concludes author Gong-Hong Wei.

Hua et al. report rs11672691 risk SNP-mediated remodelling of transcription factor binding sites in prostate cancer. Initial eQTL analysis in 471 normal primary prostate tissue samples showed that the rs 11672691 $\mathrm{G}$ risk allele is associated with decreased and increased abundance of PCAT19-short and PCAT19-long, respectively, suggesting that rs11672691 reciprocally regulates these isoforms. Interestingly, rs11672691 mapped to the promoter of the PCAT19-short isoform, which is located in the third intron of the PCAT19-long isoform. Motif analysis revealed that the risk variants of rs11672691 and rs887391 - an rs11672691 linkage disequilibrium
SNP - decreased the binding of the transcription factors homeobox protein NKX3.1 and transcriptional repressor protein YY1, respectively, to the PCAT19-short promoter. Indeed, knockdown of NKX3.1 and YY1 decreased PCAT19-short expression and, interestingly, upregulated PCAT19-long expression in prostate cancer cells. Further mechanistic experiments revealed that rs 11672691 is a bifunctional region with both PCAT19-short promoter and PCAT19-long enhancer activity. A SNP-mediated promoterenhancer switching mechanism was uncovered whereby the rs 11672691 and rs887391 risk variants decrease NKX3.1 and YY1 binding to the PCAT19-short promoter but increase PCAT19-long enhancer activity and, therefore, expression. Functional analysis revealed that PCAT19-long drives prostate cancer proliferation, migration, and invasion in vitro and exacerbates tumour growth and metastasis in vivo. Consistently, gene expression analyses in cell lines and clinical data sets revealed that PCAT19-long drives expression of a subset of cell cycle genes, an event that occurred through interaction with the heterogeneous nuclear ribonucleoprotein A/B (HNRNPAB) complex.

"We next plan to investigate the role of PCAT19 isoforms in aggressive forms of prostate cancer and assess the rs11672691 SNP combined with PCAT19 isoforms as a biomarker," adds author Housheng Hansen He.

Collectively, these findings provide a mechanistic insight into the function of the rs11672691 risk allele in prostate cancer, an understanding that could be exploited for the development of novel diagnostics or therapeutics.

Conor A. Bradley

ORIGINAL ARTICLES Gao, P. et al. Biology and clinical implications of the 19q13 aggressive prostate cancer susceptibility locus. Cell 174, 576-589 (2018) | Hua, J. T. et al. Risk SNP-mediated promoter-enhancer switching drives prostate cancer through IncRNA PCAT19. Cell 174, 564-575 (2018) 